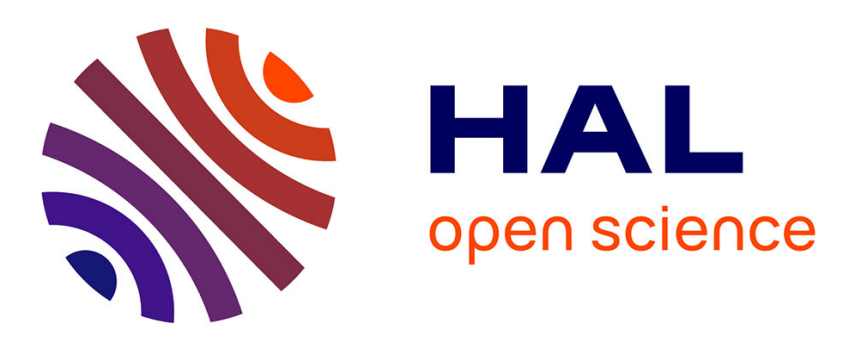

\title{
Implementation of the boundary integral method for electromagnetic scattering problems with geometrical discontinuities
}

Thierry Jacques, Laurent Nicolas, Christian Vollaire

\section{- To cite this version:}

Thierry Jacques, Laurent Nicolas, Christian Vollaire. Implementation of the boundary integral method for electromagnetic scattering problems with geometrical discontinuities. IEEE Transactions on Magnetics, 2002, 38 (2 Part 1), pp.753-756. hal-00140465

\section{HAL Id: hal-00140465 \\ https://hal.science/hal-00140465}

Submitted on 6 Apr 2007

HAL is a multi-disciplinary open access archive for the deposit and dissemination of scientific research documents, whether they are published or not. The documents may come from teaching and research institutions in France or abroad, or from public or private research centers.
L'archive ouverte pluridisciplinaire HAL, est destinée au dépôt et à la diffusion de documents scientifiques de niveau recherche, publiés ou non, émanant des établissements d'enseignement et de recherche français ou étrangers, des laboratoires publics ou privés. 


\title{
Implementation of the Boundary Integral Method for Electromagnetic Scattering Problems With Geometrical Discontinuities
}

\author{
Thierry Jacques, Laurent Nicolas, and Christian Vollaire
}

\begin{abstract}
The boundary integral method is used to solve scattering by perfect electric conducting or perfect dielectric bodies. This paper deals with different aspects of the modeling: frequency domain and time domain formulations, computing, solver. Methods allowing the treatment of geometrical discontinuity for all kinds of mesh are specially discussed. Validation of the formulations is carried by comparison with other codes.
\end{abstract}

Index Terms-Boundary element methods, discontinuities, numerical analysis.

\section{INTRODUCTION}

$\mathbf{T}$ HE APPLICATION of Maxwell's equations at a corner or at an edge shows that the components of the fields parallel to discontinuity line are bounded. On the other hand, the perpendicular components are infinite. In [1], since the tangential field is discontinuous, the functional nodes are duplicated. Semidiscontinuous elements are then successfully used, and the functional nodes are moved away from the edge. In [2], Schlemmer chooses same elements for the time domain boundary integral method and uses an interpolation between the functional node and the discontinuity line. In [3], Rego implements these elements and uses specific interpolation function. More than 24 kinds of elements are enumerated. These methods are validated using simple examples such as scattering by a cylinder or by a cube.

However, for more complex geometries such as an airplane, these methods are not satisfying. In this paper, two methods to handle geometrical discontinuities are presented for both time domain and frequency domain formulations. Numerical methods to solve the scattering problems are described as well. Comparisons with other methods are presented in order to validate the models and to show the advantages and disadvantages of each method.

\section{TIME DOMAIN FORMULATION}

As shown in Fig. 1, an incident wave is perturbed by an object of boundary $\Sigma$.

Manuscript received July 5, 2001; revised October 25, 2001

The authors are with the CEGELY, UMR CNRS 5005, Ecole Centrale de Lyon, Ecully, France (e-mail: Thierry.Jacques@eea.ec-lyon.fr; Laurent.Nicolas@eea.ec-lyon.fr; Christian.Vollaire@eea.ec-lyon.fr).

Publisher Item Identifier S 0018-9464(02)00910-X.
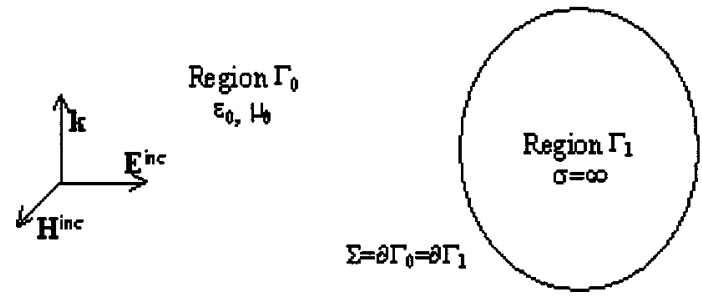

Fig. 1. Description of the problem. The conductivity $\sigma$ of a perfect electrical conductor is infinite.

\section{A. Formulation}

For a point located on the outer surface of a perfect electrical conducting, the magnetic field is computed using the following expression:

$$
\begin{array}{r}
\mathbf{H}(\mathbf{r}, t)=2 \mathbf{H}^{\mathrm{inc}}+\oiint_{S}\left(1+\frac{R}{c} \frac{\partial}{\partial t}\right) \mathbf{J}\left(\mathbf{r}-\mathbf{R}, t-\frac{R}{c}\right) \\
\times \frac{\mathbf{R}}{2 \pi R^{3}} d s
\end{array}
$$

where $\mathbf{H}^{\mathbf{i n c}}$ is the incident magnetic field, and $\mathbf{J}$ is the electric current density. This formulation has been first developed in [4] with a second-order time scheme and a constant time step $\Delta t$ :

$$
\begin{aligned}
h & =\operatorname{Int}\left(\frac{R}{2 c \Delta t}\right) \\
\theta & =\frac{R}{c \Delta t}-2 h \\
\mathbf{J}\left(\mathbf{r}, m \Delta t-\frac{R}{c}\right) & =\sum_{k=0}^{2} T_{k}(\theta) \mathbf{J}^{m-2 h-k}(\mathbf{r})
\end{aligned}
$$

where $\mathbf{J}^{m}(\mathbf{r})$ is the electric current density at the point $\mathbf{r}$ at time $m \Delta t$, and $T_{k}$ are interpolation's functions. Lagrange's polynomial functions are chosen. While Bluck uses this formulation written for the magnetic field, the unknown used in (1) is the electric current density since it requires three times less memory.

When using second-order boundary element, the following matrix system is obtained:

$$
A \mathbf{J}^{m}=2 \mathbf{n} \times \mathbf{H}^{i n c, m}+\sum_{k=1}^{p} B_{k} \mathbf{J}^{m-k}
$$

where $A$ and $B_{k}$ are sparse matrices. The $p$ matrices $B_{k}$ are not stored separately, but all the contributions of the node $j$ to 


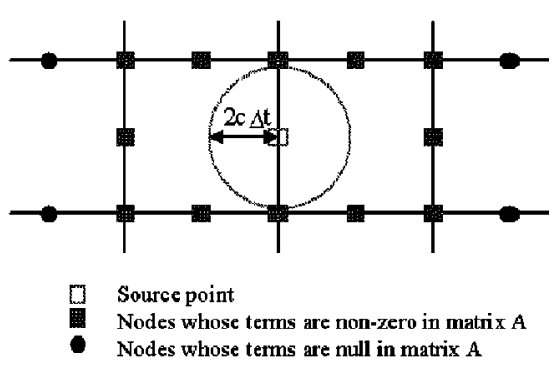

Fig. 2. Distribution of the contributions of the nodes. The contributions are equal to the integral on the surface. The center is the source point, and its radius is equal to $2 c \Delta t$
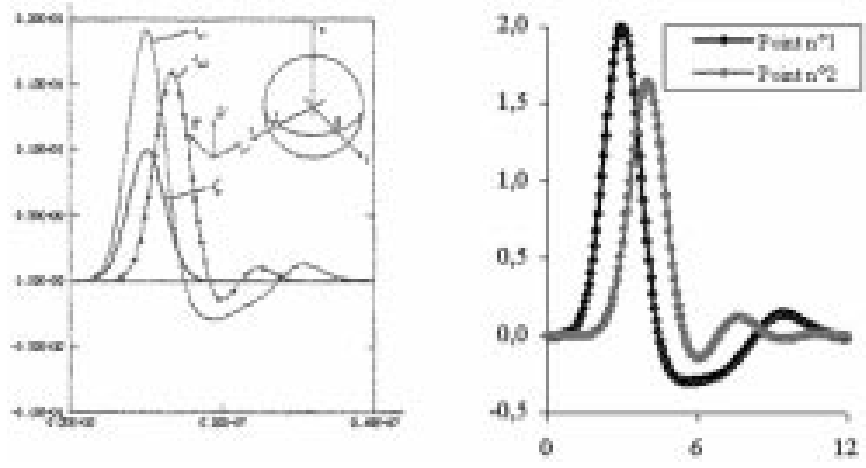

Fig. 3. Scattering of Gaussian impulse by a unit sphere. Magnetic field at equatorial plane of the sphere. Left: Schlemmer results [2]. Right: 290 nodes, 96 quadrilateral boundary elements, $\Delta x=0.075 \mathrm{~m}$.

the node $i$ are regrouped together: A time skyline storage is obtained. Regarding the matrix $A$, only the nodes located on the same elements than the source points have a nonzero contribution (Fig. 2). The contribution of the source point to itself is a 3 $\times 3$ matrix proportional to the identity.

At each time step, the biconjugated gradient algorithm with a diagonal preconditioning is used to solve the linear system. On the other hand, the solution obtained at the previous time step is used as initial vector, increasing, then, the rapidity of the convergence.

To validate this model, the scattering of Gaussian impulse by an unit sphere is solved. The results are compared with Schlemmer results [2], showing good agreement (see Fig. 3).

\section{B. Geometrical Discontinuity}

At an edge or at a corner, the normal vector is discontinuous, like the electrical current density. For an edge, on both sides of the discontinuity line, a functional node is required in order to interpolate the field on the elements. The contribution of the node on the other side of the discontinuity becomes then infinite. In order to treat the geometrical discontinuity problem, the source points are moved inside the elements at a distance equal to $\Delta x=c \Delta t$. Neither functional nor geometrical nodes are displaced. For each kind of finite element (quadrilateral or triangle), three kinds of points have to be considered, depending

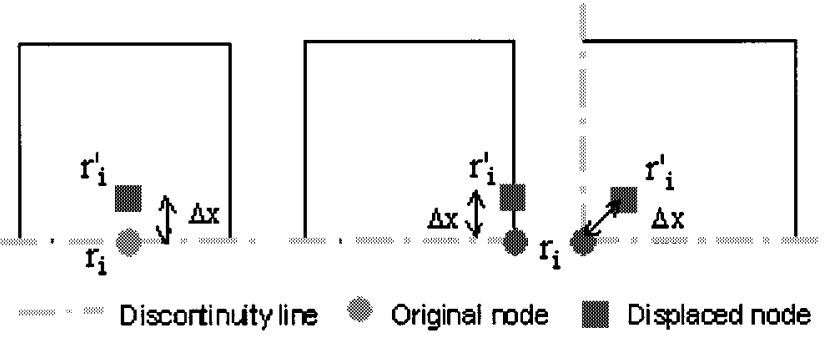

Fig. 4. Moving of source points toward the inside of boundary elements.

on the type of node (principal or secondary) and on the location of the discontinuity line.

By applying (1) at point $r_{i}^{\prime}$ and by multiplying it with the normal vector at point $\mathbf{r}_{i}$, (6) may be written at the time $t=$ $m \Delta t$ :

$$
\begin{array}{r}
\left(\alpha_{i i}-\mathbf{c}_{i i}^{0} \cdot \mathbf{n}_{i}\right) \mathbf{J}_{i}^{m}+\mathbf{n}_{i} \times \sum_{x_{q}, x_{i} \in K} \mathbf{J}_{q}^{m} \times\left(\mathbf{c}_{i q}^{0}-\alpha_{i q} \mathbf{n}_{q}\right) \\
=2 \mathbf{n} \times \mathbf{H}^{\mathbf{i n c}}\left(\mathbf{r}_{i}^{\prime}, m \Delta t\right)+\mathbf{n}_{i} \times \sum_{n} \sum_{k=1}^{m} \mathbf{J}_{n}^{m-k} \times \mathbf{c}_{i n}^{k}
\end{array}
$$

where the contributions $\mathbf{c}_{i q}^{k}$ are equal to

$$
\begin{aligned}
\mathbf{c}_{i q}^{2 m}= & \oiint_{(R / 2 \Delta x)} \mathbf{n}_{q}(s)\left(T_{0}(\theta)+\frac{R}{\Delta x} \dot{T}_{0}(\theta)\right) \frac{\mathbf{R}}{2 \pi R^{3}} d s \\
& +\oiint_{(R / 2 \Delta x)} \mathbf{n}_{q}(s)\left(T_{2}(\theta)+\frac{R}{\Delta x} \dot{T}_{2}(\theta)\right) \frac{\mathbf{R}}{2 \pi R^{3}} d s \\
\mathbf{c}_{i q}^{2 m+1}= & \oiint_{(R / 2 \Delta x)} \mathbf{n}_{q}(s)\left(T_{1}(\theta)+\frac{R}{\Delta x} \dot{T}_{1}(\theta)\right) \frac{\mathbf{R}}{2 \pi R^{3}} d s
\end{aligned}
$$

This method requires a stronger stability criterion than usual since the space step $\Delta x$ must be inferior to $\beta \Delta r$ instead of $\Delta r / \sqrt{2} . \beta$ is a real number between 0.4 and 0.48 , and $\Delta r$ is the largest inner radius of the finite elements. This decrease of time step implies that the number of right-hand side coefficients stored must be increased. On the other hand, this method may be adapted to all kinds of mesh. For a secondary node on a discontinuous boundary [Fig. 4(a)], $\alpha_{i i}$ is at least equal to 0.8. For a principal node, $\alpha_{i i}$ is larger than 0.4 [Fig. 4(b)] or 0.3 [Fig. 4(c)]. For a node on a continuous surface, $\alpha_{i i}$ is equal to 1 , and $\alpha_{i q}$ are equal to 0 . It is observed that this method slows down the convergence of the solver.

As example, the scattering of a Gaussian pulse by an unit cube has been computed. The cube is meshed with 96 quadrilateral elements and 390 nodes. The space time is equal to $5 \mathrm{~cm}$. The results are in good agreement with those of Bluck and Vechinski (see Figs. 5-7).

The method presented here is valid for perfect electric conductor. For the case of dielectric object, methods using the boundary integral method maybe found in [6] and [7]. 

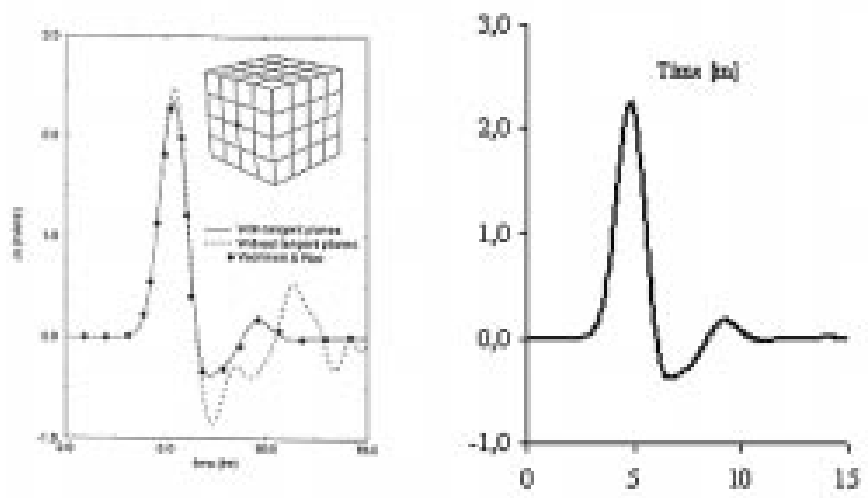

Fig. 5. Electrical current density at the point $(0,0,-0.5) . \Delta x=0.05 \mathrm{~m}$. Left: Scattering obtained by Bluck and Vechinski [5]. Right: Field obtained with our method.

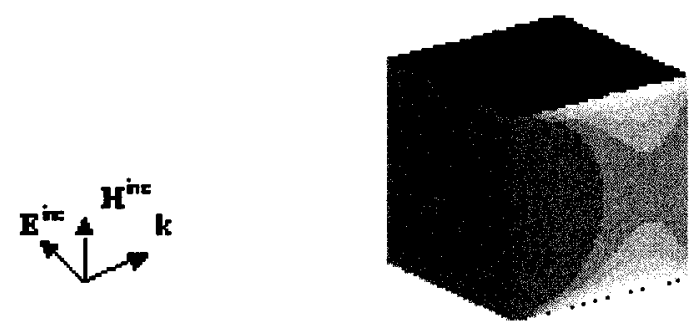

2.73

Fig. 6. Magnetic field at $t=210^{-8} \mathrm{~s}$. $\Delta t=10^{-10} \mathrm{~s}$. Scattering of a Gaussian impulse by a $1-\mathrm{m}$ side conducting cube with 798 nodes.
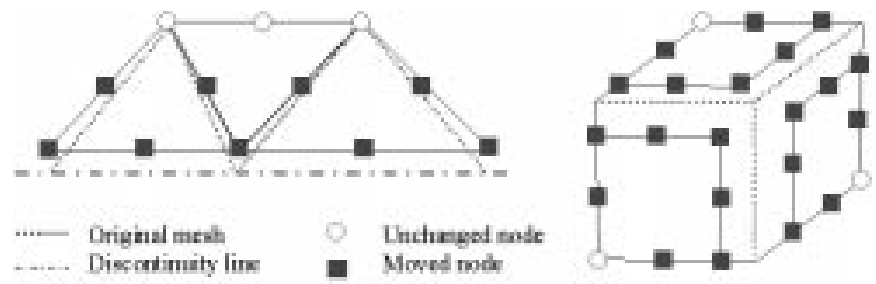

Fig. 7. Moving of functional and geometrical nodes.

\section{FREQUENCY-DOMAIN FORMULATION}

\section{A. Formulations}

By separating time and space in (1), the electric current density may be written at each point of the surface $S$ of an object considered as a perfect electrical conducting body:

$$
\mathbf{J}(\mathbf{r})=2 \mathbf{n} \times \mathbf{H}^{\mathbf{i n c}}(\mathbf{r})+2 \mathbf{n} \times \oiint_{S} \mathbf{J}(s) \times \nabla G d s
$$

where $G$ is the Green's function associated with the wave number $k=\omega / c$, and $\mathbf{n}$ is the normal unit vector to the surface $S$. For a dielectric body, the formulations are obtained by summing the integral equations written on both sides of the boundary by applying the theorem of Green. The contribution of the normal field to the tangential field (and vice versa) is equal to the difference of gradients of Green's functions. This formulation leads to 16 degrees of freedom per node corresponding to the continuous fields (electric and magnetic current density, normal magnetic flux density and normal electric flux density) on the boundary [8].

In both cases, the problem generates a full and non-Hermitian matrix. The diagonal is dominating. The obtained linear
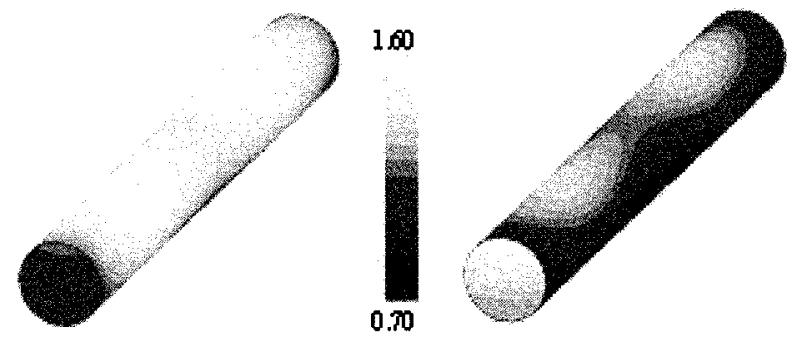

Fig. 8. Boundary element method. Magnetic and electric fields. Scattering of a $750-\mathrm{MHz}$ plane wave by a dielectric cylinder $\left(\mu_{r}=1, \varepsilon_{r}=2.47\right)$.
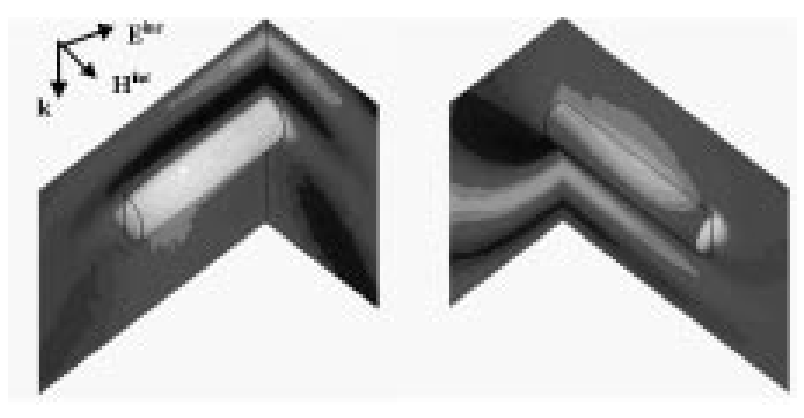

Fig. 9. Finite element method. Magnetic and electric fields. Scattering of a $750-\mathrm{MHz}$ plane wave by a dielectric cylinder $\left(\mu_{r}=1, \varepsilon_{r}=2.47\right)$. Display with one symmetry plane.

system is solved by using the BiCGStab(1) algorithm [8] with a left diagonal preconditioning. All the tested geometries have been solved using a low number of iterations corresponding to a few hundred matrix vector multiplications. For a given mesh, the algorithm shows more difficulties to converge when the frequency increases. This is due to the fact that the number of nodes per wavelength decreases, and the diagonal is less dominating. As shown in [9], ten nodes per wavelength are at least required in order to obtain a good accuracy. To take into account the geometrical discontinuities for problems in frequency domain, several solutions maybe used.

\section{B. Geometrical Discontinuities}

To get around this problem, the functional nodes are moved toward the inside of elements. Our method consists of moving the geometrical node as well. This choice is justified by the fact the distance and the surface are small in comparison with the size of the object. The computed field is dependent on the mesh close to the discontinuity. This method does not take care of all kinds of surfaces.

The scattering of a $750-\mathrm{MHz}$ plane wave by a dielectric cylinder $\left(\mu_{r}=1, \varepsilon_{r}=2.47\right)$ has been calculated by the boundary integral method and by the finite element (FE) method [10]. The length and the radius of the object are equal, respectively, to a 2.0 and a 0.25 wavelength. For the FE method, the electrical field is computed on the edge of finite elements contained in the volume $(1.5 \lambda \times 0.75 \lambda \times 0.75 \lambda)$. The magnetic field is then calculated by derivation of the electrical field, explaining the bad accuracy of the field. As shown in Figs. 8 and 9, both results are in good agreement. However, the boundary element method leads to a better accuracy than the FE method since the FE method actually sums the electric field on both sides of geometrical discontinuity. 


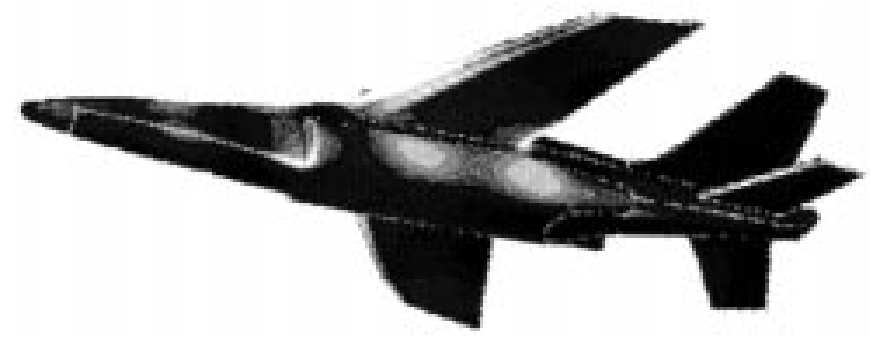

Fig. 10. Electrical current density: Scattering of $100-\mathrm{MHz}$ plane wave by an airplane.

The scattering of a 100-MHz plane wave by an airplane modelized as a perfect electric conductor has been computed on 64 processors of the Cray T3E (see Fig. 10). It is meshed with 2960 boundary elements and 62418 degrees of freedom. The maximum electrical field is located at the back of the wing of the airplane. The irregularities shown in front of the wing are related to the bad accuracy of the mesh.

\section{CONCLUSION}

In this paper, two different methods to take into account geometrical discontinuities with the boundary element method have been presented. The method developed for the time domain formulation takes into account all types of mesh. Only three kinds of points for each kind of element have to be distinguished. The stability criterion has to, however, be stronger than usual, implying an increase in the storage of previous time steps. For the frequency domain, the method is rougher, but it gives satisfying computational and numerical results.

\section{REFERENCES}

[1] M. S. Ingber and R. H. Ott, "An application of the boundary element method to the magnetic field integral equation," IEEE Trans. Antennas Propagat., vol. 39, pp. 606-611, May 1991.

[2] E. Schlemmer, W. M. Rucker, and K. R. Richter, "Calcul des champs électromagnétiques diffractés par un obstacle diélectrique tridimensionnel par éléments de frontière en régime transitoire," IEEE J. Phys. III, pp. 2115-2126, Nov. 1992.

[3] J. J. do Rêgo Silva, Ed., "Acoustic and elastic wave scattering using boundary elements," in Topics in Engineering. Boston, MA: Computat. Mech., 1994, vol. 18.

[4] M. J. Bluck, M. D. Pocock, and S. P. Walker, "An accurate method for calculation of singular integrals arising in time-domain integral equation analysis of electromagnetic scattering," IEEE Trans. Antennas Propagat., vol. 45, pp. 1793-1798, Dec. 1997.

[5] M. J. Bluck and S. P. Walker, "Time-domain BIE analysis of large three dimensional electromagnetic scattering problems," IEEE Trans. Antennas Propagat., vol. 45, pp. 894-901, May 1997.

[6] E. Schlemmer, W. M. Rucker, and K. R. Richter, "Boundary element computations of 3D transient scattering from lossy dielectric objects," IEEE Trans. Magn., vol. 29, pp. 1524-1527, Mar. 1993.

[7] M. D. Pocock, M. J. Bluck, and S. P. Walker, "Electromagnetic scattering from 3-D curved dielectric bodies using time-domain integral equations," IEEE Trans. Antennas Propagat., vol. 46, pp. 1212-1219, Aug. 1998.

[8] T. Jacques, L. Nicolas, and C. Vollaire, "Parallelization of the boundary element method for the modeling of large electromagnetic scattering problems," in Proc. PARELEC, Trois-Rivières, QC, Canada, Aug. $27-30,2000$.

[9] G. L. G. Sleijpen and D. R. Fokkema. (1993, Sept.) BiCGStab(1) for linear equations involving unsymmetric matrices with complex spectrum. ETNA [Online], pp. 11-32. Available: http://www.math.ruu.nl/ people/sleijpen.

[10] C. Vollaire, L. Nicolas, and A. Nicolas, "Finite elements coupled with absorbing boundary conditions on parallel distributed memory computer," IEEE Trans. Magn., vol. 33, pp. 1448-1451, Mar. 1997.

[11] S. P. Walker and C. Y. Leung, "Parallel computation of time-domain integral equation analyzes of electromagnetic scattering and RCS," IEEE Trans. Antennas Propagat., vol. 45, pp. 614-619, Apr. 1997. 